\title{
Plant protein influence on appetite and food intake in healthy subjects
}

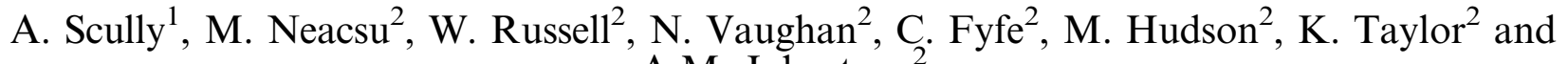 \\ A.M. Johnstone ${ }^{2}$ \\ ${ }^{1}$ University College Dublin, Belfield, Dublin 4, Ireland and ${ }^{2}$ Rowett Institute of Nutrition and Health, University of \\ Aberdeen, AB25 2ZD, UK.
}

Both the World Health Organisation (WHO) and World Cancer Research Fund UK (WCRFUK) promote increasing the population's consumption of legumes and pulses alongside reduction in red meat, for preventative nutrition in gut health ${ }^{(1,2)}$. There is growing interest in the implementation of a plant-based diet, particularly in the context of high protein diets for appetite control ${ }^{(3)}$. Recent short-term meal feeding studies highlight the role for vegetable protein in influencing satiety ${ }^{(4)}$, through dietary fibre and protein effects. However, there is a paucity of data on appetite control to compare plant proteins.

One of the main aims of this dietary intervention study was to evaluate motivation to eat, assessed as a within-subject design during a controlled diet trial. We recruited 20 healthy subjects (6 male, 14 female) age $23-63 \mathrm{yr}$ with BMI $19 \cdot 3-38.9 \mathrm{~kg} / \mathrm{m}^{2}$ to participate in a randomised, cross-over human dietary intervention study. Ethics was obtained from The Rowett Institute Research Committee and volunteers provided written informed consent. Subjects recorded habitual intake by weighed food diary for the initial baseline week of the study. The plant diets were buckwheat and fava bean vegetarian diets, with all food provided for the 7 day dietary intervention period and there was a 1 week wash out between treatments. Participants recorded motivation to eat during waking hours with a subjectively reported paper visual analogue scale (VAS) with questions to assess hunger, fullness, prospective consumption and desire to eat. Appetite was assessed by combining 4 questions 'How hungry do you feel?', 'How full do you feel?', 'How strong is your desire to eat?', and 'How much do you think you could eat now?'. Post-meal VAS indicated satiety and pleasantness of the meals. The formula '(desire to eat + hunger + (100- fullness) + prospective consumption)/4' was used to calculate average appetite score for each diet ${ }^{(5)}$.

Based on the habitual food diaries, volunteers consumed $22.9 \pm 5.6 \mathrm{~g}$ fibre prior to the intervention diets. This significantly increased to $44.8 \pm 6.3 \mathrm{~g}$ and $37.1 \pm 5.7 \mathrm{~g}$ respectively, during the fava and buckwheat diets $(\mathrm{p}<0.001)$. ANOVA confirmed that there was no significant effect of diet type on motivation to eat or appetite, relative to baseline diets (see Figure 1). Furthermore, there was no significant difference between the two intervention diets on appetite.

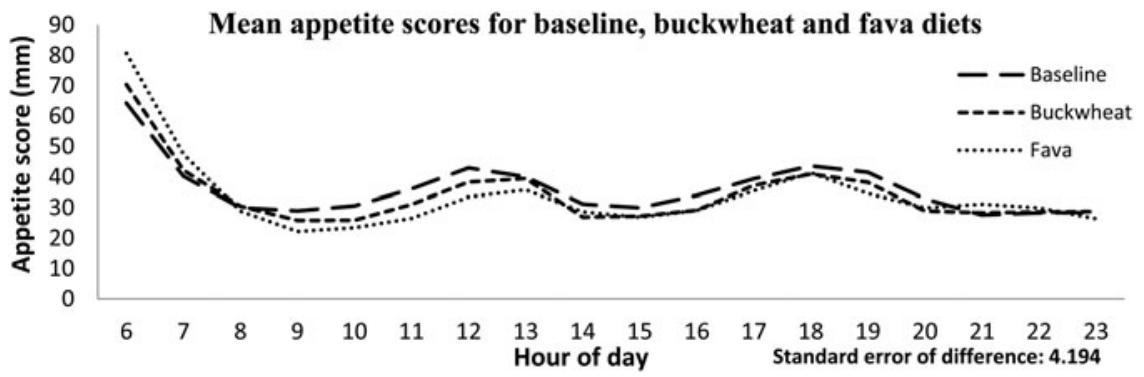

Fig. 1.

These data confirm that plant protein, consumed as buckwheat and fava bean rich diets, can easily be incorporated into the diet in the short term to maintain protein and fibre intake and diet quality without compromising appetite control.

1. Wcrf-uk.org. (2016) Plant foods and cancer prevention World Cancer Research Fund UK http://www.wcrf-uk.org/uk/preventing-cancer/ ways-reduce-cancer-risk/plant-foods-and-cancer-prevention.

2. WHO, Q\&A on the carcinogenicity of the consumption of red meat and processed meat (2016) http://www.who.int/features/qa/cancer-red-meat/en/.

3. Kristensen MD, Bendsen NT, Christensen SM, Astrup A, Raben A Food Nutr Res. 2016 Oct 19:60:32634.

4. Neacsu M, Fyfe C, Horgan G, Johnstone AM. Am J Clin Nutr. 2014 Aug;100(2), 548-58

5. Anderson G, Catherine N, Woodend D, Wolever T. Am J Clin Nutr 2002 Nov;76(5), 1023-30. 